\title{
Novel bonding patterns and optoelectronic properties of the two-dimensional $\mathrm{Si}_{x} \mathrm{C}_{y}$ monolayers
}

\author{
Dong Fan, ${ }^{1}$ Shaohua $\mathrm{Lu},{ }^{1,}$ 网 Yundong Guo, ${ }^{2}$ and Xiaojun $\mathrm{Hu}^{1,{ }^{1}}$ \\ ${ }^{1}$ College of Materials Science and Engineering, Zhejiang University of Technology, Hangzhou 310014, China \\ ${ }^{2}$ School of Engineering and Technology, Neijiang Normal University, Neijiang, 641000, China.
}

(Dated: December 28, 2016)

\begin{abstract}
The search of new two-dimensional (2D) materials with novel optical and electronic properties is always desirable for material development. Here, we report a comprehensive theoretical prediction of 2D SiC compounds with different stoichiometries from C-rich to Si-rich. Besides the previously known hexagonal SiC sheet, we identified two types of hitherto-unknown structural motifs with distinctive bonding features. The first type of 2D SiC monolayer, including $t$-SiC and $t-\mathrm{Si}_{2} \mathrm{C}$ sheet, can be described by tetragonal lattice. Among them, $t$-SiC monolayer sheet is featured by each carbon atom binds with four neighboring silicon atoms in almost the same plane, constituting a quasi-planar four-coordinated rectangular moiety. More interestingly, our calculations demonstrate that this structure exhibits a strain-dependent insulator-semimetal transition, suggesting promising applications in strain-dependent optoelectronic sensors. The second type of $2 \mathrm{D} \mathrm{SiC}$ sheet is featured by silagraphyne with acetylenic linkages(-C $\equiv \mathrm{C}-$ ). Silagraphyne shows both high pore sizes and Poisson's ratio. These properties make them a potentially important material for applications in separation membranes and catalysis. Moreover, one of the proposed structures, $\gamma$-silagraphyne, is a direct-band-gap semiconductor with a bandgap of $0.89 \mathrm{eV}$, which has a strong absorption peak in the visible-light region, giving a promising application in ultra-thin transistors, optical sensor devices and solar cell devices.
\end{abstract}

Since the demonstration of the first isolated graphene sheet in 2004, 2D atomic crystals have received much attention. For graphene, due to its many extraordinary properties, it has potential applications in a wide range of areas. However, the pristine graphene is a gapless semi-metal, which means that it is difficult to control the number of carriers. This dramatically limits its applications in the field effect transistor, photovoltaic cell, and etc. Thus, the subject of finding new 2D materials beyond graphene is one of the most active fields of current material research. These research include graphyne, single-layer hexagonal boron nitride $(h$-BN), [1] transition metal oxides or chalcogenides, [2],[3] phosphorene, [4] as well as group IVVI and IIIV layered crystals.[5] Particularly, besides graphene and graphyne, a strong research topic in group-IV 2D elemental monolayers have sprung up in recent years. However, these group-IV 2D elemental derivatives show the properties of Dirac fermion behavior without spin-orbit coupling, which create a set of challenges for application in conventional electronic devices due to the lack of band gap at the Fermi level.

2D SiC have recently emerged as a promising material with tunable band gaps for potential applications in optoelectronics and electronics.11 Especially, inspired by the successful syntheses of the graphene-like hexagonal $\mathrm{SiC}$ sheet in experiment, 12 a few carbon-rich $\mathrm{SiC}$ monolayers, such as para$\mathrm{SiC}_{3},[6] g-\mathrm{SiC}_{2},[7]$ and $p t-\mathrm{SiC}_{2},[8]$ were predicted at one particular stoichiometry. Among all of these newly structures, $g-\mathrm{SiC}_{2}$ sheet, a direct band gap of $1.09 \mathrm{eV}$ is nearly ideal material for flexible optoelectronic devices. Recently, using first-principle calculations coupled with the cluster expansion method, Shi et al. reported a structural search on $\mathrm{SiC}$ sheets with different stoichiometry.[9] However, their structure search is limited to graphene-like hexagonal lattice. Therefore, in order to further explore the configuration space and unique properties of $\mathrm{SiC}$ sheets, a global structure search with both variable lattice type and stoichiometry is required.
In this work, we report a range of low-energy $\mathrm{Si}_{x} \mathrm{C}_{y}$ monolayers with distinguished bonding patterns and electronic properties using unbiased particle swarm optimization (PSO) structure search algorithm.[10] The flat hexagonal lattices have been successfully predicted as the low-energy structures for previously reported $g-\mathrm{SiC}_{2}, \mathrm{SiC}$ and many other $\mathrm{Si}_{x} \mathrm{C}_{y}$ monolayers in this work. Based on the comprehensive analysis and simulations, we find 2D tetragonal silagraphene named $t$-SiC and $t-\mathrm{Si}_{2} \mathrm{C}$ sheets with both thermodynamic and mechanical stability. The $t$-SiC exists a buckled tetragonal atomic arrangement formed by strong $\mathrm{C} 2 \mathrm{p}-\mathrm{Si} 2 \mathrm{p}$ bonding with an out-of-plane electron delocalization. More importantly, a phase transition from direct band gap semiconductor to semi-metal is observed when biaxial tensile strain is exerted on $t$ - $\mathrm{SiC}$ monolayer, suggesting promising applications in strain-dependent optoelectronic sensors. Moreover, six different silagraphyne sheets with higher pore sizes and Poisson's ratio are found, making them potential candidates for separation membranes and catalytic materials. Especially for $\gamma$-silagraphyne, it exhibits direct band gap and obvious optical absorption in visible-light spectrum, indicating a desirable material in microelectronics, solar cell materials and nanoscale optical sensors devices.

Candidate structures were obtained by global structural optimization method as implemented in CALYPSO code.[10] It has been successfully applied to various crystal surfaces and low dimensional materials. [11-13] Structure search was performed with the concentration of carbon ranges from 0.167 to 0.833 . The subsequent structural relaxation and total energy calculations were carried out using the density functional theory (DFT) in applying general gradient approximation (GGA) in the Perdew-Burke-Ernzerhof (PBE) [14] parameterization for exchange correlation potential as implemented in the Vienna $a b$ initio simulation package (VASP).[15] The plane wave cut off energy was set to $650 \mathrm{eV}$. The convergence 


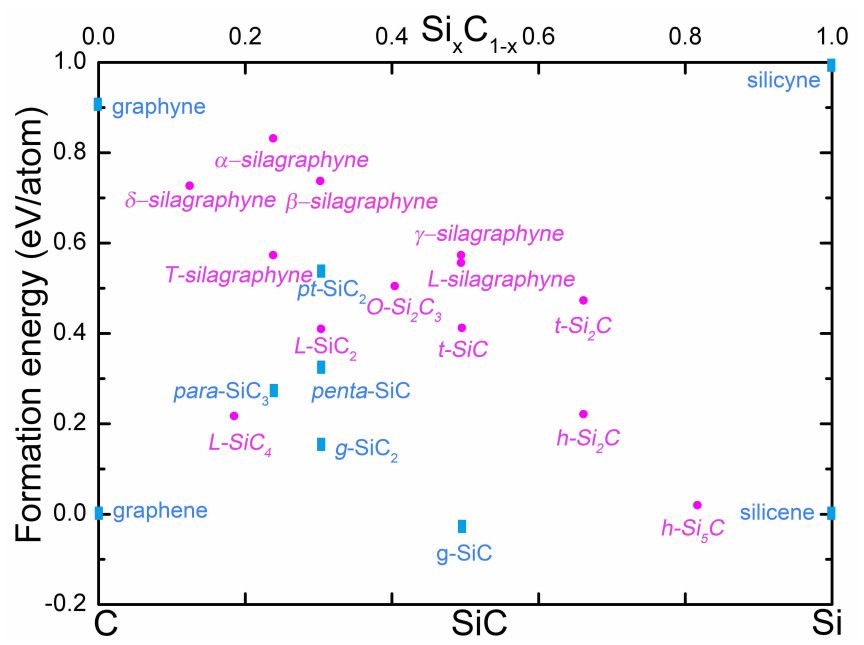

Figure. 1. Schematic diagram of the calculated formation energies of $\mathrm{Si}_{x} \mathrm{C}_{y}$ sheets under conditions from C-rich to Si-rich. Previously known para-SiC ${ }_{3},[6] g-\mathrm{SiC}_{2},[7] p t-\mathrm{SiC}_{2}[8]$ and penta-SiC are also presented for comparison.

criterion of self-consistent calculations for ionic relaxations was $10^{-5} \mathrm{eV}$ between two consecutive steps and the atomic positions and unit cells were optimized until the atomic forces were less than $0.01 \mathrm{eV} / \AA$ without any symmetry constraints. A slab model containing a $20 \AA$ vacuum region in the normal direction was selected to simulate isolated 2D materials. Z-axis was fixed during structure relaxation. HeydScuseriaErnzerhof (HSE06) functional[16] was used to calculate band structures of selected configurations. In order to study the dynamical stability of the proposed structures, we performed phonon calculations and ab initio molecular dynamics (AIMD) simulations. Phonon calculations were conducted by the density functional perturbation theory (DFPT) method using the PHONOPY code.[17] AIMD simulations were carried out in the NVT ensemble with a time step of $1 \mathrm{fs}$ for a total time of $5 \mathrm{ps}$.

We collect structures with the lowest energies at each stoichiometric composition and calculate the formation energy $\left(\mathrm{E}_{f}\right)$ to evaluate the relative stabilities of the predicted $2 \mathrm{D} \mathrm{SiC}$ compounds, which is defined as follows:

$$
E_{f}=\left(E_{S i x C y}-x \mu_{S i}-y \mu_{C}\right) /(x+y)
$$

where $\mathrm{E}_{f}$ denotes the formation energy of the corresponding $2 \mathrm{D} \mathrm{SiC}$ compounds, $\mathrm{E}_{S i}, \mathrm{E}_{C}$ and $\mathrm{E}_{S i x} \mathrm{C}_{y}$ are the total energy of a single $\mathrm{Si}$ atom, a single $\mathrm{C}$ atom, and the $2 \mathrm{D} \mathrm{Si}_{x} \mathrm{C}_{y}$ compound.

Fig 1 shows the schematic diagram of the calculated formation energies of $\mathrm{Si}_{x} \mathrm{C}_{y}$ sheets under conditions from C-rich to Si-rich. Previously known sheets, such as hexagonal $\mathrm{SiC}$, para- $\mathrm{SiC}_{3}, g-\mathrm{SiC}_{2}$, and $p t-\mathrm{SiC}_{2}$ have been successfully reproduced in this work, and our calculated formation energies are consistent with previous results. Besides, two types of hitherto unknown structure motifs are revealed in the global structure search. The predicted low-energy structures of several al- lotropes with unique bonding patterns and the corresponding lattice parameters are listed in Table S1. All 2D compounds with novel bonding geometries are predicted and plotted in Fig 2, S1 and S2. Moreover, kinetic stability of these 2D sheets is verified by both phonon spectrum calculations and AIMD simulations. Next, we perform a systematic analysis of the chemical bonding, mechanical and electronic properties on the newly found structures.

Fig 2a displays the geometric configuration of $t$-SiC monolayer. Its rectangular lattice constants are $\mathrm{a}=3.67 \AA$ and $\mathrm{b}$ $=3.21 \AA$, with a thickness $\mathrm{c}=1.01 \AA$. One unit cell of $t$-SiC consists of $2 \mathrm{C}$ atoms and $2 \mathrm{Si}$ atoms, respectively. Especially, all the atoms in the supercell bond to four neighboring atoms. Usually, just like graphene, the majority of 2D sheets have three-fold coordinated bonding moieties. All C and $\mathrm{Si}$ atoms in the $t$-SiC sheet are quasi-planar tetracoordinated atoms. To the best of our knowledge, this is the first time to report pure tetracoordinated carbon atoms in $2 \mathrm{D}$ covalent materials. The stabilizing mechanisms and bonding characteristics are disclosed by analyzing the deformation electron density data, which is defined as the total electronic density of 2D SiC system excluding the electronic densities of isolated $\mathrm{Si}$ and $\mathrm{C}$ atoms. Deformation charge density of $t$-SiC monolayer is shown in Fig S3, revealing that some electrons are extracted from $\mathrm{Si}$ atoms and delocalized around the four $\mathrm{Si}-\mathrm{C}$ bonds. This is crucial to stabilizing the quasi-planar tetracoordinated moieties. The buckling of $\mathrm{t}-\mathrm{SiC}$ monolayer binds $\mathrm{Si}-\mathrm{C}$ bonds in tetragonal lattice and weakens interaction between two neighboring atoms. In order to gain more insights into chemical bonding in $t$-SiC sheet, we plot the electron localization function (ELF) to determine the extent of covalent character. An isosurface of ELF for $t$-SiC is illustrated in Fig $\mathrm{S} 4$, with an isovalue of 0.80 au, indicating the formation of strong $\mathrm{Si}-\mathrm{C} \sigma$ bonds.
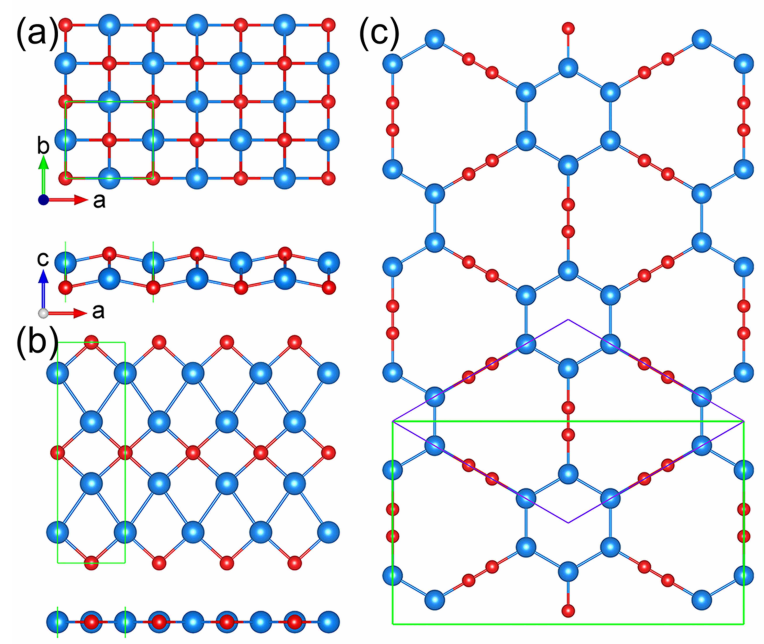

Figure. 2. Top (upper) and side (lower) views of the structure of (a) $t$-SiC, (b) $t$ - $\mathrm{Si}_{2} \mathrm{C}$ and (c) $\gamma$-silagraphyne monolayer. The blue and red balls represent $\mathrm{Si}$ and $\mathrm{C}$ atoms, respectively. The rhombohedra (violet) and rectangular (green) unit cells are presented. 
Another structure (named as $t-\mathrm{Si}_{2} \mathrm{C}$ ) with tetragonal lattice is also revealed. As shown in Fig $2 \mathrm{~b}, t-\mathrm{Si}_{2} \mathrm{C}$ monolayer forms the exactly planar structure and the optimized lattice constants for $t-\mathrm{Si}_{2} \mathrm{C}$ monolayer are $\mathrm{a}=2.79 \AA$ and $\mathrm{b}=9.09 \AA$. Fig S3(b) shows the electron transfer from $\mathrm{Si}$ atoms to $\mathrm{C}$ atoms in $t$ $\mathrm{Si}_{2} \mathrm{C}$ monolayer. Moreover, carbon $2 \mathrm{p}$ electrons are delocalized around the four $\mathrm{Si}-\mathrm{C}$ bonds in the same plane, suggesting the covalent character of $\mathrm{Si}-\mathrm{C}$ bonds. For $t-\mathrm{Si}_{2} \mathrm{C}$, its ELF isosurfaces exhibit two domains: one is the distribution around the centered four $\mathrm{Si}-\mathrm{C}$ bonds symmetrically, and the other is the $\mathrm{Si}$-Si dimer rows, whereas the bonding between $\mathrm{Si}$ atoms is weak compared with $\mathrm{Si}-\mathrm{C} \sigma$ bonds.

Besides tetragonal silagraphene, we also demonstrate a new 2D $\mathrm{SiC}$ monolayer with acetylenic linkages $(-\mathrm{C} \equiv \mathrm{C}-)$, silagraphyne, namely. Fig 2 and S1 display three 2D SiC compounds, silagraphyne, containing acetylenic linkages that are more stable than graphyne by comparing their formation energies. It is worth noting that the shortest distances between sp-carbon atoms are 1.24 and $1.23 \AA$ of $\alpha(\beta)$-silagraphyne and $\gamma$-silagraphyne, respectively. This indicates that the localization of electron density is in the binding regions. As demonstrated in Fig S1 and S2, all the structures of silagraphyne, except for L- and $\gamma$-silagraphyne with a buckling of 0.65 and $0.48 \AA$, are purely planar. It is clear to see that the acetylenic linkages generate pores, which are much larger than those in graphene and graphyne.[18] According to our calculations, $\alpha-, \beta$ - and $\gamma$-silagraphyne have the inverse of the specific surface area of $0.362,0.432$ and $0.537 \mathrm{mg} / \mathrm{m}^{2}$, respectively. This size of the pore is very important in the desalination of sea water,[19] photocatalyst, lithium-ion battery and energy storage, [4] because they have high porosity and high specific surface area compared to graphyne (i.e., $0.379 \mathrm{mg} / \mathrm{m}^{2}$ for $\alpha$ graphyne, $0.461 \mathrm{mg} / \mathrm{m}^{2}$ for $\beta$-graphyne and $0.582 \mathrm{mg} / \mathrm{m}^{2}$ for $\gamma$-graphyne).[18] The calculative ELF maps of silagraphyne monolayers are demonstrated in Fig S5. It's clear to see that all of the ELFs of C-C and $\mathrm{Si}-\mathrm{C}$ bonds are localized at the bond center, similar to the ELF maps in $t$-SiC monolayer.

The stability of the $2 \mathrm{D} \mathrm{Si}_{x} \mathrm{C}_{y}$ sheet can first be understood by calculating its formation and cohesive energies, as shown in Fig 1 and Table S1. The results show that newly predicted $t$-SiC is higher in energy than graphene by $0.39 \mathrm{eV} /$ atom, but lower than that of previously reported $p t-\mathrm{SiC}_{2}$ by 0.19 $\mathrm{eV} /$ atom. Simultaneously, the cohesive energy of $t$-SiC and $t$ $\mathrm{Si}_{2} \mathrm{C}$ is 5.55 and $4.85 \mathrm{eV} /$ atom, respectively, which are larger than that of silicene $(3.93 \mathrm{eV} /$ atom $)$ and lower than that of graphene $(7.94 \mathrm{eV} /$ atom). Thus, it is proved that $2 \mathrm{D}$ tetragonal $\mathrm{SiC}$ monolayer has higher structural stability and stronger binding energy comparative with silicene. Obviously, the silagraphyne monolayers are metastable while they all have lower formation energies with respect to graphene and silicene. In particular, we also note that silagraphyne monolayers are energetically preferable over experimentally confirmed graphdiyne films. [8] This implies that the newly found silagraphyne sheets might be synthesized in the experiment. Moreover, we calculated the cohesive energy to evaluate the stability of proposed silagraphyne structures. As shown in Ta- ble S1, the cohesive energies of silagraphyne monolayers are ranging from 4.50 to $6.55 \mathrm{eV} /$ atom, which are significantly lower than that of graphene, but still higher than that of silicene, attesting that the silagraphyne sheets are energetically stable.
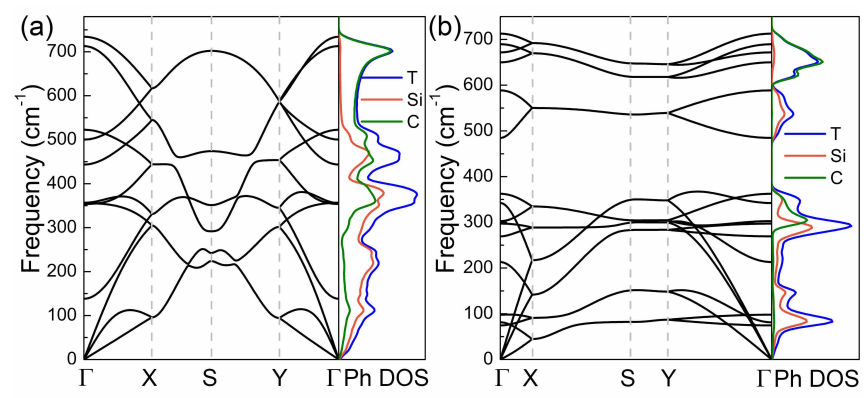

Figure. 3. Phonon band dispersions (left panel) and partial PhDOS (right panel) of (a) $t$-SiC and (b) $t$-Si C monolayer are calculated by linear response theory. $\Gamma(0,0,0), \mathrm{X}(1 / 2,0,0), \mathrm{S}(1 / 2,1 / 2,0)$ and $\mathrm{Y}(0$, $1 / 2,0$ ) refer to special points in the first Brillouin zone of reciprocal space. There is no imaginary frequency in the whole path.

To examine the dynamic stability of proposed structures, the phonon dispersion spectra and phonon density of states ( $\mathrm{Ph}$ DOS) were calculated by using DFPT method along the high-symmetry lines, as shown in Figure 3 and Figure S6. It is observed that there is no imaginary phonon frequency in the entire Brillouin zone, confirming that tetragonal silagraphene sheets are dynamically stable. For $t-\mathrm{SiC}$ and $t-\mathrm{Si}_{2} \mathrm{C}$ monolayer, we note that the highest frequency reaches up to 735 and $713 \mathrm{~cm}^{-1}$, respectively, which is slightly higher than that of silicene $\left(580 \mathrm{~cm}^{-1}\right)[20]$ and $\mathrm{MoS}_{2}$ monolayer (473 $\left.\mathrm{cm}^{-1}\right),[21]$ indicating robust $\mathrm{Si}-\mathrm{C}$ bonds in these 2D systems. Analysis of partial PhDOS reveals that the highest frequency of $t$ - $\mathrm{SiC}$ is mainly originated from $\mathrm{C}$ atoms. The phonon dispersion curves of $t-\mathrm{Si}_{2} \mathrm{C}$ indicate the presence of phonon gap and have the separation of acoustic and optical branches with the maximum vibrational frequency at $\sim 368 \mathrm{~cm}^{-1}$. Detailed analysis of the PhDOS reveals that the lower acoustic modes are associated with the constituent of Si atoms, while the high frequency vibrational modes attribute to the $\mathrm{Si}-\mathrm{C}$ stretching modes. To further understand the stability of these unique structures at ambient conditions, we have performed AIMD simulations within the canonical NVT ensemble at $1000 \mathrm{~K}$ for $5 \mathrm{ps}$ with a time step of $1 \mathrm{fs}$. Total energy development of simulation time and snapshots taken at the end of each time is shown in Figure S7-S9. During the final NVT simulation, $t$-SiC and $t-\mathrm{Si}_{2} \mathrm{C}$ sheets are stable up to a temperature of 800 $\mathrm{K}$, while other silagraphene and silagraphyne can maintain the structural integrity at $1000 \mathrm{~K}$ (Figure S8, S9). The above results reveal that these $2 \mathrm{D} \mathrm{SiC}$ monolayers exhibit a high thermal stability.

For a mechanically stable 2D material, the elastic constants just have to fulfill the following requirements:[22] $\mathrm{C}_{11} \mathrm{C}_{22}$ $\mathrm{C}_{12} \mathrm{C}_{21}>0$ and $\mathrm{C}_{66}>0$. For silagraphene, the calculated elastic constants satisfy this formula, and the calculated $\mathrm{C}_{66}$ 
is positive (Table S2), indicating that all the proposed silagraphene sheets are mechanically stable. The in-plane stiffness modulus, which can be derived from the elastic constants by: $\mathrm{E}=\left(\mathrm{C}_{11} \mathrm{C}_{22}-\mathrm{C}_{12} \mathrm{C}_{21}\right) / \mathrm{C}_{11}$. Tetragonal silagraphene sheets present elastic in-plane stiffness with 119 and $169 \mathrm{GPa} \cdot \mathrm{nm}$ along the b direction for $t-\mathrm{SiC}$ and $t-\mathrm{Si}_{2} \mathrm{C}$, respectively, which are more than double of that of silicene. The calculated elastic constants of six silagraphyne monolayers also satisfy the mechanical stability criteria, indicating that the 2D silagraphyne monolayers are mechanically stable (Table S3). Interestingly, the calculated in-plane stiffness values for three kinds of silagraphyne going from $\alpha$-silagraphyne, to $\beta$ - and $\gamma$-silagraphyne are $17.00,26.33$ and $47.55 \mathrm{GPa} \cdot \mathrm{nm}$, respectively. The in-plane stiffness increases with decreasing specific surface area and is significantly lower than that of graphyne. Thus, these 2D sheets inserting acetylenic linkages are much softer than graphyne and graphene, due to the lower planar packing densities of these structures with respect to the graphene. Similar to graphyne, $\alpha-, \beta$ - and $\gamma$-silagraphyne have Poissons ratios between 0.5 and 1 , suggesting a higher structural deformation along the perpendicular direction to the plane.[18]

Figure 4 shows the band structures (calculated via HSE functional) of $t$-SiC and $t$-SiC structures. As presented in Figure $4 \mathrm{a}, t-\mathrm{SiC}$ is a direct band gap semiconductor with a band gap of $1.84 \mathrm{eV}(0.85 \mathrm{eV}$ at the PBE level) at the $\Gamma$ point. Its valence band maximum (VBM) is contributed by the $\mathrm{Si}-3 \mathrm{~s}$ $(\sigma)$ orbitals, while the conduction band minimum (CBM) is contributed by Si-3p $(\pi)$ and C-2p $(\pi)$ orbitals (Figure S10). However, different from $t$-SiC, our band structure calculations show that $t-\mathrm{Si}_{2} \mathrm{C}$ is metallic with a finite density of states at the Fermi energy. Figures $4 \mathrm{c}(\mathrm{e})$ and $4 \mathrm{~d}(\mathrm{f})$ indicate the highest occupied orbital (HOMO) and the lowest unoccupied orbital (LUMO) at the $\Gamma$ point, respectively. It is interesting to note that, for $t-\mathrm{Si}_{2} \mathrm{C}$, the HOMO clearly shows the strong bonding between two $\mathrm{Si}$ atoms, while LUMO is mainly distributed around the Si atoms.

Supplementary Figure S11 shows the phonon dispersion for $t$-SiC sheet when biaxial strain is applied, for strain values of $1 \%, 9 \%$ and $-9 \%$. There is not any imaginary phonon mode in phonon dispersion, so its strained monolayer is also stable. Subsequently, for $t$-SiC sheet, we calculate the HSE band structure at different biaxial strain (Figure 4g). Notably, we find that the band structure can be transformed from direct band gap to semimetal at a relatively suitable biaxial strain $(10 \%)$. The direct band gap persists under a tensile strain of 1 to $4 \%$ and the band gap decreases from 1.72 to $1.31 \mathrm{eV}$. According to Shockley-Queisser limit,[23] for single-gap photovoltaic devices with the band gap of $1.34 \mathrm{eV}$, a maximum photoelectric conversion efficiency of $33.7 \%$ can be achieved. Thus, the direct band structure of small strained monolayer supplies a favorable advantage for the applications in photoelectric devices. Figure 5a and Figure S12 demonstrate the electronic band structures of a few selected silagraphyne monolayer calculated with PBE functional. It is observed that $\alpha$-silagraphyne and $\beta$-silagraphyne show the presence of
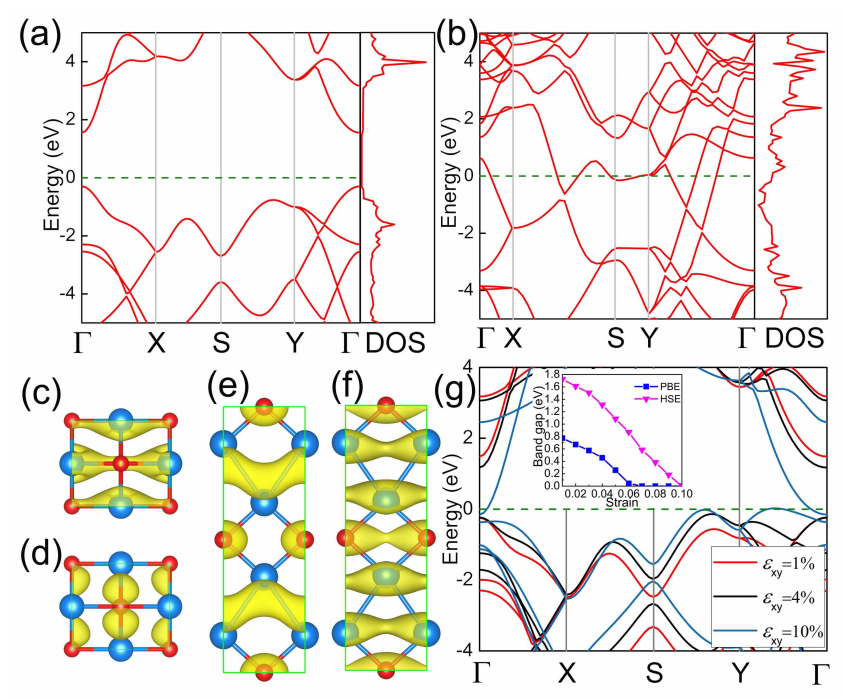

Figure. 4. Electronic band structure and DOS of (a) $t$-SiC and (b) $t$ - $\mathrm{Si}_{2} \mathrm{C}$ structures are calculated by using HSE06 functional. Banddecomposed charge density distributions are illustrated in $\mathrm{c}$ to f. (c) The highest occupied electronic state and (d) the lowest unoccupied electronic state of $t$-SiC at the $\Gamma$ point. (e) The highest occupied electronic state and (f) the lowest unoccupied electronic state of $t$-Si C at the $\Gamma$ point. The Fermi level is set to zero and marked by the dashed lines. (g) Band structure of $t$-SiC with different biaxial strains. Inset shows the band gap of monolayer as a function of biaxial strains (calculated by PBE and HSE06 functionals).

Dirac points at the Fermi level, but they show different number and location of Dirac points. $\alpha$-silagraphyne has Dirac points located at $\mathrm{K}$ high symmetry points, while $\beta$-silagraphyne exists Dirac points in the $\Gamma-\mathrm{M}$ direction. These interesting results are similar to the electronic structure of graphyne. However, $\gamma$-silagraphyne and T-silagraphyne show direct band gaps, where the CBM and VBM are located at the $M$ and $\Gamma$ points in the Brillouin zone, respectively. Using the HSE functional, we note that $\gamma$-silagraphyne and T-silagraphyne have the band gap of 0.89 and $2.02 \mathrm{eV}$, respectively, while $\delta$-silagraphyne and L-silagraphyne show semimetal property.

Band gap plays a fundamental role in semiconductor materials, due to the strong dependence of band gap and solar cells energy conversion efficiency. The characteristics of moderate direct band gap for 2D SiC monolayers make it promising solar-cell absorption materials. Thus, optical absorption spectra of predicted low energy structures were calculated at the HSE06 level. For comparison, calculations were also carried out for silicon. Our results for the silicon curves are in accord with previous calculations.[24] Figure 5b shows the optical absorption spectra. According to the spectral range, we separated the absorption spectrum into three parts, namely the infrared, visible, and ultraviolet regions, respectively. Analogous to silicon, due to the large direct band gap, $t$-SiC monolayer can absorb photons in the ultraviolet range mostly. Surprisingly, $\gamma$-silagraphyne can absorb the sunlight at lower energies than that of $t$-SiC sheet, implying that this structure can 

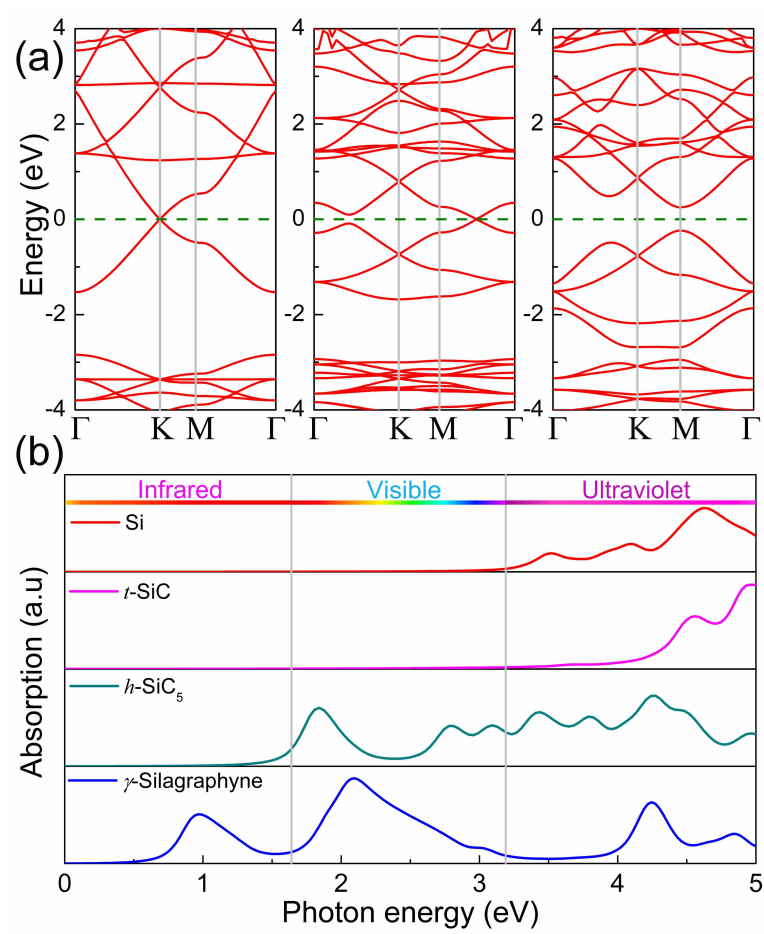

Figure. 5. (a) Calculated band structure of $\alpha$-silagraphyne (left), $\beta$ silagraphyne (middle), and $\gamma$-silagraphyne (right) monolayers. The Fermi level is set to zero and marked by dashed lines. (b) Optical absorption coefficient of the proposed structures, silicon data is presented for comparison. (calculated by HSE06 functional).

capture more sunlight and make the efficient use of solar energy compared to silicon.

The aforementioned results suggest that tetragonal silagraphene and silagraphyne are very promising candidates for application in future optoelectronic nanodevices due to their unique structural and electronic properties. Thus, it is desirable to synthesize these new monolayers experimentally. Compared to the honeycomb structure, tetragonal lattice seems impossible according to the valence electron pair repulsion rule.[25] However, in fact, planar tetracoordinated carbon has been first proposed by Hoffman et al. (1970)[26] and later synthesized in metal compound molecules (1977).[27] In organic chemistry, the compound having the shape of a square pyramid is called [3.3.3.3] fenestrane (or pyramidane), consisting of a central carbon atom which serves as a common vertex for four fused carbocycles. Experimentally, although this chemical compound has not been synthesized yet, the synthesis of related $\mathrm{Ge}_{2}\left[\mathrm{C}_{4}\left(\mathrm{SiMe}_{3}\right)_{4}\right]$ and $\mathrm{Sn}\left[\mathrm{C}_{4}\left(\mathrm{SiMe}_{3}\right)_{4}\right]$ have been reported.[28] Recent theoretical results also elaborate the existence of planar 4-coordinated $\mathrm{C}$ atoms in 2D materials, such as 2D BC compounds,[12] $t$-TiC[29] and pentagraphene[30] et al. Thus, in consideration of the chemical similarity between carbon and silicon, it provides a possibility to produce a newly tetragonal silagraphene experimentally.

Here, for $t$-SiC monolayer, a possible strategy to obtain (a)
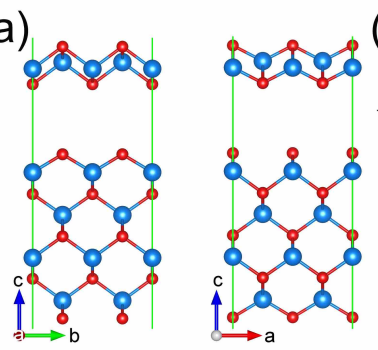

(b)

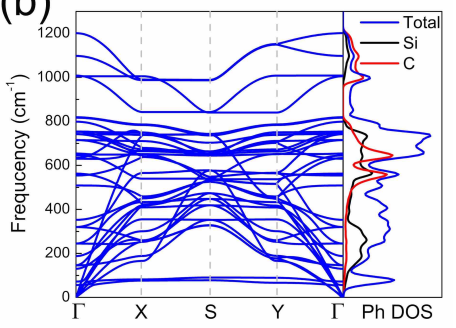

(c)

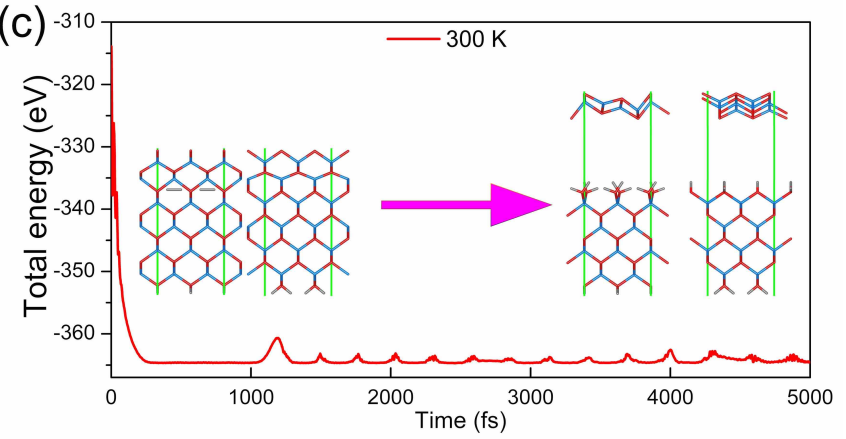

Figure. 6. (a) Structure of 3C-SiC (0001) surface containing t-SiC monolayer and corresponding Phonon spectrum (b). (c) The calculated energy evolution of hydrogen in intercalated 3C-SiC surface during AIMD simulation at $300 \mathrm{~K}$. The insets show the initial and final structures viewed along $\mathrm{a}$ and $\mathrm{b}$ direction, respectively.

this sheet by hydrogen intercalation to break the subsurface $\mathrm{C}$-Si covalent bonds in $3 \mathrm{C}-\mathrm{SiC}$ is proposed. We first isolate $t$ $\mathrm{SiC}$ sheet from 3C-SiC substrate (Optimized structural model is shown in Figure 6a) and check its structural stability by phonon calculation (Figure 6b). Next, the effect of hydrogen intercalation in the subsurface of $3 \mathrm{C}-\mathrm{SiC}$ for stripping a $t-\mathrm{SiC}$ monolayer is investigated by performing the AIMD simulation shown in Figure 6c. After a period of time, the system has reached thermodynamic equilibrium, a $t$-SiC monolayer with buckled structure is observed and striped from the 3C$\mathrm{SiC}$ film surface. Although this process may have high energy barriers, there is an experimental evidence that hydrogen/deuterium can selectively interact with $3 \mathrm{C}-\mathrm{SiC}$ substrate at subsurface by using high-resolution electron energy loss spectroscopy and synchrotron radiation based photo-emission spectroscopy techniques. [31]

In conclusion, we find two types of $2 \mathrm{D} \mathrm{SiC} \mathrm{monolayers}$ with unique bonding structures: tetragonal silagraphene and silagraphyne. The proposed tetragonal silagraphene sheets ( $t$-SiC and $t$-SiC) exhibit a novel planar (quasi) tetracoordinated $\mathrm{Si}-\mathrm{C}$ pattern, while the silagraphyne shows an acetylenic linkages ( $s p$ carbon atoms) in the 2D crystal lattice. Then, kinetics and dynamics stability of the predicted monolayers were investigated by performing AIMD simulations and the calculations of phonon dispersion curves, respectively. Especially, for $t$-SiC monolayer, each $\mathrm{Si}$ atom is coordinated to four $\mathrm{C}$ atoms to form a tetragonal motif, which closely resembles pyramidane in structures with an approximate $\mathrm{D} 2 \mathrm{~h}$ symmetry. To our knowledge, such monolayer purely con- 
sists of quasi-planar covalent tetracoordinated carbon and silicon atoms have never been reported in the previous literature. Owing to its unique atomic patterns and strain-induced semiconductor to metal transition properties, $t$-SiC shows promise for applications in optoelectronic sensors when the suitable biaxial tensile strain is applied. Silagraphyne sheets have higher pore sizes, Poisson's ratio, and tunable band gap energies varying from 0.00 to $2.02 \mathrm{eV}$. These structural and electronic properties make silagraphyne as alternative materials for special applications that need softer materials and the next-generation photovoltaic devices. Finally, as a theoretical exploration, we propose a fabrication process by using hydrogen intercalation to break the subsurface $\mathrm{C}$-Si covalent bonds in 3C-SiC. In view of distinct bonding structure and dynamics stability to endure the biaxial strain, these novel structures might be synthesized experimentally and used to fabricate photoelectric conversion device with a high efficiency.

This work was supported by the National Natural Science Foundation of China (Grant Nos.50972129, 50602039, and 11504325), and Natural Science Foundation of Zhejiang Province (LQ15A040004). This work was also supported by the international science technology cooperation program of China (2014DFR51160).

*1sh@zjut.edu.cn

$\dagger$ huxj@zjut.edu.cn

[1] Kenji Watanabe, Takashi Taniguchi, and Hisao Kanda. Directbandgap properties and evidence for ultraviolet lasing of hexagonal boron nitride single crystal. Nature materials, 3(6):404409, 2004.

[2] Ping He, Haijun Yu, Haoshen Zhou, et al. Layered lithium transition metal oxide cathodes towards high energy lithium-ion batteries. Journal of Materials Chemistry, 22(9):3680-3695, 2012.

[3] Haotian Wang, Hongtao Yuan, Seung Sae Hong, Yanbin Li, and Yi Cui. Physical and chemical tuning of two-dimensional transition metal dichalcogenides. Chemical Society Reviews, 44(9):2664-2680, 2015.

[4] Likai Li, Yijun Yu, Guo Jun Ye, Qingqin Ge, Xuedong Ou, Hua Wu, Donglai Feng, Xian Hui Chen, and Yuanbo Zhang. Black phosphorus field-effect transistors. Nature nanotechnology, 9(5):372-377, 2014.

[5] H Şahin, S Cahangirov, M Topsakal, E Bekaroglu, E Akturk, R Tugrul Senger, and Salim Ciraci. Monolayer honeycomb structures of group-iv elements and iii-v binary compounds: First-principles calculations. Physical Review B, 80(15):155453, 2009.

[6] Yi Ding and Yanli Wang. Geometric and electronic structures of two-dimensional sic3 compound. The Journal of Physical Chemistry C, 118(8):4509-4515, 2014.

[7] Liu-Jiang Zhou, Yong-Fan Zhang, and Li-Ming Wu. Sic2 siligraphene and nanotubes: novel donor materials in excitonic solar cells. Nano letters, 13(11):5431-5436, 2013.

[8] Yafei Li, Fengyu Li, Zhen Zhou, and Zhongfang Chen. Sic2 silagraphene and its one-dimensional derivatives: where planar tetracoordinate silicon happens. Journal of the American Chemical Society, 133(4):900-908, 2010.
[9] Zhiming Shi, Zhuhua Zhang, Alex Kutana, and Boris I Yakobson. Predicting two-dimensional silicon carbide monolayers. ACS nano, 9(10):9802-9809, 2015.

[10] Yanchao Wang, Jian Lv, Li Zhu, and Yanming Ma. Crystal structure prediction via particle-swarm optimization. Physical Review B, 82(9):094116, 2010.

[11] Shaohua Lu, Yanchao Wang, Hanyu Liu, Mao-sheng Miao, and Yanming Ma. Self-assembled ultrathin nanotubes on diamond (100) surface. Nature communications, 5, 2014.

[12] Xinyu Luo, Jihui Yang, Hanyu Liu, Xiaojun Wu, Yanchao Wang, Yanming Ma, Su-Huai Wei, Xingao Gong, and Hongjun Xiang. Predicting two-dimensional boron-carbon compounds by the global optimization method. Journal of the American Chemical Society, 133(40):16285-16290, 2011.

[13] Li-Ming Yang, Vladimir Ba?i?, Ivan A Popov, Alexander I Boldyrev, Thomas Heine, Thomas Frauenheim, and Eric Ganz. Two-dimensional cu2si monolayer with planar hexacoordinate copper and silicon bonding. Journal of the American Chemical Society, 137(7):2757-2762, 2015.

[14] John P Perdew, Kieron Burke, and Matthias Ernzerhof. Generalized gradient approximation made simple. Physical review letters, 77(18):3865, 1996.

[15] Georg Kresse and Jürgen Furthmüller. Efficient iterative schemes for ab initio total-energy calculations using a planewave basis set. Physical review B, 54(16):11169, 1996.

[16] Jochen Heyd, Gustavo E Scuseria, and Matthias Ernzerhof. Hybrid functionals based on a screened coulomb potential. The Journal of Chemical Physics, 118(18):8207-8215, 2003.

[17] Atsushi Togo and Isao Tanaka. First principles phonon calculations in materials science. Scripta Materialia, 108:1-5, 2015.

[18] Antonio Ruiz Puigdollers, Gerard Alonso, and Pablo Gamallo. First-principles study of structural, elastic and electronic properties of $\alpha-, \beta$-and $\gamma$-graphyne. Carbon, 96:879-887, 2016.

[19] Minmin Xue, Hu Qiu, and Wanlin Guo. Exceptionally fast water desalination at complete salt rejection by pristine graphyne monolayers. Nanotechnology, 24(50):505720, 2013.

[20] Seymur Cahangirov, Mehmet Topsakal, Ethem Aktürk, $\mathrm{H}$ Şahin, and Salim Ciraci. Two-and one-dimensional honeycomb structures of silicon and germanium. Physical review letters, 102(23):236804, 2009.

[21] Alejandro Molina-Sanchez and Ludger Wirtz. Phonons in single-layer and few-layer mos 2 and ws 2. Physical Review $B, 84(15): 155413,2011$.

[22] Richard Charles Andrew, Refilwe Edwin Mapasha, Aniekan M Ukpong, and Nithaya Chetty. Mechanical properties of graphene and boronitrene. Physical Review B, 85(12):125428, 2012.

[23] William Shockley and Hans J Queisser. Detailed balance limit of efficiency of p-n junction solar cells. Journal of applied physics, 32(3):510-519, 1961.

[24] Yaguang Guo, Qian Wang, Yoshiyuki Kawazoe, and Puru Jena. A new silicon phase with direct band gap and novel optoelectronic properties. Scientific reports, 5, 2015.

[25] William L Jolly. Modern inorganic chemistry. McGraw-Hill College, 1984.

[26] Roald Hoffmann, Roger W Alder, and Charles F Wilcox Jr. Planar tetracoordinate carbon. Journal of the American Chemical Society, 92(16):4992-4993, 1970.

[27] F Albert Cotton and Michelle Millar. The probable existence of a triple bond between two vanadium atoms. Journal of the American Chemical Society, 99(24):7886-7891, 1977.

[28] Vladimir Ya Lee, Yuki Ito, Akira Sekiguchi, Heinz Gornitzka, Olga A Gapurenko, Vladimir I Minkin, and Ruslan M Minyaev. Pyramidanes. Journal of the American Chemical Society, 
135(24):8794-8797, 2013.

[29] Zhuhua Zhang, Xiaofei Liu, Boris I Yakobson, and Wanlin Guo. Two-dimensional tetragonal tic monolayer sheet and nanoribbons. Journal of the American Chemical Society, 134(47):19326-19329, 2012.

[30] Shunhong Zhang, Jian Zhou, Qian Wang, Xiaoshuang Chen, Yoshiyuki Kawazoe, and Puru Jena. Penta-graphene: A new carbon allotrope. Proceedings of the National Academy of Sci- ences, 112(8):2372-2377, 2015.

[31] Patrick Soukiassian, Erich Wimmer, Edvige Celasco, Claudia Giallombardo, Simon Bonanni, Luca Vattuone, Letizia Savio, Antonio Tejeda, Mathieu Silly, Marie D?angelo, et al. Hydrogen-induced nanotunnel opening within semiconductor subsurface. Nature communications, 4, 2013. 